\title{
Influencia entre conductas sociales y decisiones empresariales
}

\author{
Vicente SÁNCHEZ JIMÉNEZ \\ Universidad Complutense de Madrid \\ vicentes@pdi.ucm.es
}

Recibido: 03-12-2014

Aceptado: 25-03-2015

\section{Resumen}

Al adentrarnos en el estudio del papel que están jugando en la actualidad las empresas en la conformación social debemos resaltar que esta materia propia del marco de la microeconomía, en concreto de las interrelaciones entre empresa y sociedad, es el ejemplo práctico de los vínculos que se producen en un marco más amplio, el socioeconómico. Es en este último, donde se establece para su mejor compresión la necesidad de profundizar en el encuentro entre las decisiones económicas que se producen en el seno de las empresas y las motivos sociales que las condicionan, de la misma forma que la influencia que las conductas empresariales establecen en el conjunto de la sociedad. Es decir, lo que podríamos entender como uno de los nexos más importantes entre sociedad y economía. El presente artículo se introduce en estas cuestiones con la pretensión de poder encontrar las interconexiones entre las exigencias sociales y la orientación de las empresas ante las mismas. De la misma forma, que intentar aclarar que entraña lo que se ha venido denominado "ética empresarial".

Palabras clave: sociedad, empresas, ética, responsabilidad social, decisiones morales. 


\title{
Influence between Social Behavior and Business Decisions
}

\begin{abstract}
As we move into the study of the role they are currently playing in social enterprises must emphasize that this conformation own stuff under microeconomics, specifically the relationships between business and society, is the practical example of the links occur in a broader framework, socioeconomic. It is in the latter, which is set for better understanding the need to deepen the encounter between the economic decisions that occur within business and social reasons that condition, in the same way that the influence behaviors enterprise established in the whole society. That is, what might be understood as one of the most important links between society and economy. This article introduces these issues with the aim of finding the interconnections between social demands and the orientation of business to them. In the same way, which involved trying to clarify what has been called "business ethics".
\end{abstract}

Keywords: Society; Business; Ethics; Social Responsibility; Moral Decisions.

\section{Referencia normalizada}

SÁNCHEZ JiMÉNEZ, Vicente (2015): "Influencia entre conductas sociales y decisiones empresariales”, Politica y Sociedad, 52 (2), pp. 443-464.

Sumario: 1. Introducción. 2. La influencia entre el devenir social y la empresa. 3. La ética empresarial. 4. La responsabilidad de las decisiones empresariales con la sociedad. 5. Bibliografía 


\section{Introducción}

Ha sido Amartya Sen (2009), con su Teoría de la Justicia, quien ha retomado la interacción existente entre estas dos realidades, en este caso, como perfectamente indica el título de la obra, desde el estudio de la justicia. El autor bengalí diferencia en su texto lo que considera las dos líneas filosóficas de pensamiento en torno a la teoría de la justicia y que han influido, además, en la actual concepción de la idea de justicia en el ámbito de la Ciencia Económica. Por una parte, cita a Thomas Hobbes, John Locke, Jean-Jacques Rousseau y Kant, cuyo enfoque contractualista ha penetrado de forma dominante en la filosofía política contemporánea a través del libro de John Rawlz, Teoría de la Justicia escrito en 1971. Por la otra, engloba a Adam Smith, Concordet, Karl Marx y John Stuart Mill como precursores, de alguna manera, de la "teoría de la elección social" que en el siglo pasado desarrolló Kenneth Arrow. Entre esta dualidad, Sen encuentra más idóneo el segundo grupo de pensamiento, con las matizaciones adecuadas, para responder a las preguntas sobre la "mejora de la justicia y la eliminación de la injusticia en el mundo".

Con las reflexiones del profesor de Harvard volvemos desde la Economía actual al camino de las preguntas clásicas de esta Ciencia, como la distribución de la riqueza o el papel de las empresas en la sociedad. De esta forma tan gráfica, somos testigos de cómo se está produciendo en ciertos núcleos de pensamiento un cambio en la mentalidad de los economistas, los cuales han enriquecido sus investigaciones con un mayor número de variables. El propio Sen (1989:45) había denunciado un progresivo debilitamiento de la Economía como ciencia ya en los años 80 del pasado siglo, como así corroboran sus palabras: "de hecho, en la economía moderna, es precisamente la reducción de la amplia visión smitheana de los seres humanos lo que pueda considerarse como una de las mayores deficiencias de la teoría económica contemporánea. Este empobrecimiento se encuentra íntimamente relacionado con el distanciamiento de la economía y de la ética”. Opinión compartida por Fabián Estapé (1990:181), quien en una mayor concreción hacia la microeconomía, campo en el encontramos el objeto del presente artículo, la ética empresarial, mantiene que "un economista sin contacto directo con la realidad de su tiempo, sin un conocimiento por sumario que sea de los procesos de producción de su tiempo. Sin una familiaridad con ese doble mundo de motivaciones complejas que es el de los empresarios y el de los obreros, podrá realizar tal o cual aportación, pero con la mayor frecuencia edificará sobre el vacío".

Prueba de este cambio sustancial en la visión de los economistas sobre las variables que influyen en las decisiones empresariales es la extensa literatura aparecida en las últimas décadas, especialmente en esta primera década del siglo XXI. Los estudios sobre la relación entre empresa y sociedad acerca de la responsabilidad que las primeras deben mantener con respecto al resto de actores sociales se basan en la influencia recíproca entre las tres instituciones tradiciones: Estado, sociedad civil y empresa; otorgando a cada uno de ellos unos componentes bien definidos y delimitados, base de la interacción de las tres estructuras. 
En el caso del Estado encontramos un sistema que continúa amoldado a los principios decimonónicos de la conformación social: el poder ejecutivo, legislativo y judicial, en todos sus ámbitos territoriales. En cambio para la sociedad civil podemos comprobar la presencia de nuevos elementos si lo comparamos con una visión clásica de la sociedad. De esta forma a las instituciones académicas, asociaciones empresariales, organizaciones sindicales y la propia Iglesia en el caso de los países de nuestro entorno, debemos sumar las llamadas organizaciones no gubernamentales, como son las dedicadas al desarrollo social, la protección de la naturaleza, la igualdad entre hombres y mujeres,...

En los países donde la conformación de las nuevas instituciones nacidas de la sociedad civil ha producido un profundo cambio al que se está viendo abocado el conjunto de la sociedad, incluidas las empresas. Ello, además se ve fortalecido por el proceso de globalización acelerado en estos años, especialmente el de los medios de comunicación, lo que ha permitido a los ciudadanos una mayor cantidad de información en tiempo real y, por tanto, una interpretación diferente de la responsabilidad de la empresa con la sociedad. De hecho, es en los países con economías más desarrolladas y sociedades más avanzadas, donde los consumidores muestran una mayor presión hacia las decisiones empresariales.

\section{La influencia entre el devenir social y la empresa}

Siguiendo estos principios podemos apreciar cómo la llamada pérdida de confianza hacia los actores del mercado global y su capacidad de autocontrol, en particular en las empresas financieras por ser éstas las más liberalizadas, ha supuesto un importante retroceso en la concepción que la sociedad moderna occidental tenía sobre sí misma. En este sentido ha surgido una verdadera "crisis de confianza" que ha traspasado las fronteras de la economía, puesto que como afirma el Premio Nobel Kenneth Arrow (1974:77) "la confianza, la lealtad y la veracidad son exterioridades, son bienes, son mercancías, que tienen un valor real, práctico y económico".

Si en los últimos años se ha considerado al capital social como un elemento básico en la conformación del progreso económico ello es debido a la importancia que se le otorga como estructurador de la sociedad. Ambas variables, estructura social y desarrollo económico, se encuentran íntimamente ligadas, pues sin la estabilidad de la primera se considera poco viable la realización de la segunda. Ello se debe a que comparten como característica básica uno de los factores que permiten la existencia de capital social, las redes sociales, por cuanto éstas posibilitan tanto el beneficio individual de los sujetos como la consecución de fines colectivos.

Las redes sociales se han fortalecido en las últimas décadas, a la par que la sociedad de la información, especialmente aquellas que se configuran a raíz de los valores morales insertos en cada sociedad. Este crecimiento, cuya base radica en el asociacionismo 
que parte de la sociedad civil, se ha producido en aquellos países que cuentan con un modelo social más desarrollado y que podríamos considerar con mayores reservas de capital social, es el caso de los países del llamado primer mundo. En estas democracias los valores sociales se han desarrollado en una gran diversidad de campos de actividad, como son, por citar los más destacados: la lucha por la igualdad efectiva entre mujeres y hombres, la defensa de las condiciones laborales, la potenciación del ecologismo como valedor de un desarrollo sostenible, y las actuaciones de ayuda y cooperación internacional. De esta forma se han visto potenciadas por un número mayor de integrantes las organizaciones de ayuda al tercer mundo, las que dirigen sus acciones a la protección medioambiental y las de reivindicación de la igualdad entre géneros. De la misma manera el crecimiento afiliativo a los sindicatos se ha mantenido en constante progresión en los últimos años, siendo éstos una de las formas de asociación más extendidas de las sociedades actuales. Organizaciones sindicales que cuentan, además, con un espacio social muy determinado, ocupando uno de los tres pilares de las relaciones sociales, junto con las empresas y los gobiernos.

La dinámica de creación de organizaciones sociales es fiel reflejo de las variaciones que se originan en la estimación de las prioridades morales del conjunto de una sociedad y, por ende, de los valores predominantes en cada momento. La realidad del asociacionismo horizontal no es ajena al resto de instituciones, como son las Administraciones Públicas, los gobiernos y las empresas. Ello se debe a dos motivos básicos. El primero es que todas las instituciones, sin importar su naturaleza, están compuestas por individuos que se desarrollan en sociedad, participan de sus redes sociales, sus creencias e ideologías; sujetos activos en la toma de unas decisiones que, en mayor o menor medida, se impregnan de la dinámica social. El segundo se debe a que las instituciones tienen mayor aceptación social, en la medida que satisfacen las necesidades de los ciudadanos, de forma que deben velar porque los bienes y servicios que ponen a disposición de la sociedad sean potencialmente aceptables desde la perspectiva moral.

Para el caso de las empresas, estos factores tienen una importancia evidente, si tenemos en cuenta que deben orientar sus objetivos a cubrir las demandas de los individuos y de las sociedades en las que se encuentran inmersas, a la vez obtienen beneficios. Las empresas, en el marco económico actual, han de tomar a diario decisiones que les permitan sobrevivir en un mercado cada vez más abierto y globalizado, especialmente si hablamos de las empresas multinacionales que operan en la mayor parte de las economías del mundo, siendo influenciadas e influyendo sobre las poblaciones en donde están afincadas. Es decir, modifican y a la vez son modificadas por el entorno donde operan, pues han de adaptarse a la realidad de los países, ya sea donde producen o donde venden. Esta retroalimentación entre empresa y sociedad hace que los economistas preocupados por las imbricaciones entre economía y ética den carta de naturaleza a estos nexos. Así lo atestigua el sentir de Amartya Sen (1989) para quien los códigos éticos de los empresarios y profesionales son parte de los recursos productivos de la sociedad. 
Ante este doble intercambio entre empresas y sociedad, la presión que se deriva del manejo de información por parte de la opinión pública es cada vez mayor; la obtención de una mayor cantidad y calidad de información es fruto, según la teoría del capital social, de un entramado más denso de sus redes sociales, así como de la madurez cívica de sus ciudadanos. El capital social potencia de forma paralela la obtención de conocimiento y la toma de decisiones colectivas, convirtiéndose en el equilibrio de las decisiones del resto de las instituciones, como las que parten de los gobiernos ejecutores cuando hablábamos de desarrollo económico. En un mundo globalizado no sólo las empresas se expanden por más países, sino que con ellas también lo hacen las redes sociales, que, bajo valores morales, intentan vigilar sus actuaciones.

Estos movimientos van conformando el mercado económico global y la distribución de ideas y valores, principalmente de las sociedades del llamado primer mundo, por cuanto son sus empresas las que se propagan por el resto de naciones. Esta expansión económica de los países más avanzados, también de sus conocimientos, es la base para aquellos que ven en este camino un desarrollo global más armónico, como así defiende Herskovits (1952), para quien "la difusión de la ciencia y la investigación pueden ser el propósito de una visión solidaria del desarrollo a escala internacional".

La cada vez mayor vinculación entre empresa y sociedad se debe a que ambas comparten un mismo actor, las personas, que potencia tanto la democratización social como el desarrollo económico a través de sus decisiones. Es por ello que la Economía vuelve a buscar respuestas en las áreas de trabajo que comparte con otras ciencias sociales, y así la antropología económica nos plantea el cuestionamiento del ser humano como principio y protagonista de la mecánica económica y social. Por ello, el campo de trabajo antropológico es cada vez más necesario para el economista, pues su estudio es el ser humano en el marco de la sociedad y cultura a la que pertenece, y, al mismo tiempo, como producto de éstas. La Antropología analiza, entre otras cuestiones, los modos de comportamiento sociales a través del tiempo y el espacio.

La sociedad evoluciona y podemos decir que hay una nueva sociedad en cada tiempo. Nuevos valores, anhelos e ideologías donde las personas se desarrollan, aunque a la vez traigan consigo su herencia cultural. La diferencia entre las estructuras pasadas y presentes de la sociedad se encuentra en las características particulares de la misma en cada periodo de tiempo, pues el entheos, o habitus en término bourdianos, sigue siendo el mismo originario. Así, el desarrollo acumulativo es el que posibilita las variaciones de una sociedad evolutiva. Dicho en términos de la antropología del desarrollo y con palabras de Arthur Lewis (1958:459), "el desarrollo significa contar con más medios, y por tanto con más posibilidades de elección entre distintos bienes, o entre distintas dedicaciones de ocio y negocio, de tiempo libre y tiempo de trabajo".

Al fin y al cabo de lo que estamos hablando es de la concepción que la teoría económica convencional tiene sobre el comportamiento racional identificado como el comportamiento real. Para ello grosso modo podemos sintetizar este posicionamiento me- 
diante los dos métodos predominantes, por una parte, el que considera la racionalidad como la consistencia interna en la elección, por la otra, el que identifica la racionalidad con la maximización del propio interés, en lo que se conoce como la teoría del egoísmo.

Estaríamos nuevamente intentando resolver una de las cuestiones básicas de la Ciencia Económica desde los autores clásicos: la elección ante diferentes opciones. La cuestión se encuentra en saber si es una pluralidad de motivaciones o exclusivamente el egoísmo lo que mueve a los seres humanos. Es decir, utilizando términos smithianos, si nos movemos por valores como la humanidad, la justicia, la generosidad y el espíritu público por pretender ser las cualidades más beneficiosas para los demás $\mathrm{y}$, por ende, para nosotros mismos, o más bien nos movemos para satisfacer nuestras necesidades personales. De esta forma volvemos la vista a las cuestiones éticas de la motivación humana sobre cómo se debe vivir, pero más aún, cómo se debe hacer para conseguir la evolución del logro social. Por lo que denotamos que las interacciones entre capital social y economía son mayores que las simples variables que conforman el primero permitiendo el desarrollo del segundo.

Las decisiones individuales, la conformación del capital social, el beneficio individual y colectivo, el estudio de la economía y la ética no es algo que parta de los nuevos avances teóricos del conjunto de las ciencias sociales sino que se encuentra en el entheos común y único del conocimiento científico a la par que humano. Prueba de esta afirmación la encontramos en las palabras de Aristóteles (1980) en su Ética Nicomaquea, con las que cerramos éste artículo, cuando al hablar de conseguir lo bueno de manera individual apuntaba algunas características colectivas: "si bien merece la pena alcanzar este fin únicamente para un hombre, es mejor y más divino alcanzarlo para una nación o para las ciudades-estado".

Observamos cómo se ha producido una reorientación teórica y conceptual con respecto al papel que tradicionalmente se les había otorgado a las empresas. El nuevo contrato entre empresa y sociedad demanda a las empresas que lleven a cabo sus actividades observando los valores sociales y dando respuesta a las prioridades de la sociedad. Esta orientación incide en la capacidad de las corporaciones para dar respuesta a las expectativas sociales, resultando entonces que la filosofía misma de la organización y la aptitud gerencial poseen un papel trascendental en la definición de cómo debe la empresa atender las presiones y demandas de la comunidad y lograr la satisfacción de sus necesidades más urgentes. Es decir, conseguir la minimización del coste social producido por los efectos de las actividades perjudiciales para la sociedad. Por tanto, no sólo se está pidiendo que las empresas tengan buenas prácticas que respalden un comportamiento ético en sus negocios o incluso actuaciones de acción social y filantrópicas, sino además que incorporen códigos de conducta en la dirección estratégica de la compañía. Se pretende que los directivos asimilen la interrelación entre sus decisiones empresariales y consecuencias sociales, o lo que es igual, la idea de que tienen una responsabilidad social y de que su obligación es cumplirla. 
Desde la perspectiva de la teoría empresarial, la responsabilidad social se constituye como una parte más dentro del marco más amplio que es la ética de los negocios. Comprobamos como en el marco de la administración de las empresas se ha modificado la visión de las relaciones que éstas deben tener para conseguir sus fines. Se ha pasado de una misión exclusivamente económica a una socioeconómica con una cada vez más influencia de responsabilidad moral con la sociedad. Prueba de ello es la relevancia que la teorización sobre la responsabilidad social empresarial ha adquirido en los últimos años, más aún si tenemos en cuenta que es a partir de la segunda mitad del pasado siglo cuando se empieza a cuestionar el objetivo económico de las empresas como único fin y se toma en consideración el impacto de las actuaciones en la sociedad.

La exigencia de la sociedad en torno a los derechos humanos, la protección del medio ambiente, la lucha contra la corrupción, la defensa del trabajo digno, entre otros, obliga a las empresas a considerar la ética en sus decisiones. Por este motivo pasamos a analizar la llamada ética empresarial.

\section{La ética empresarial}

Aunque no es nuestra intención profundizar en el análisis de lo que se conoce como ética, pues esto nos obligaría a realizar un pormenorizado estudio de sus relaciones y vínculos con la moral, sí creemos conveniente conocer su etimología y, particularmente, su contextualización del mismo en nuestro ámbito de estudio.

La palabra ética proviene del latín ethǐcus, y este del griego $\dot{\eta} \theta \imath \kappa o ́ s$, o transcrito a nuestro alfabeto, "êthicos", cuyo significado sería carácter o modo de ser. Diferenciándolo del término latino mores, que da origen a la palabra moral y de significado costumbre. Para el Diccionario de la Lengua Española la palabra ético tiene diferentes aproximaciones, las cuales son:

1. adj. Perteneciente o relativo a la ética.

2. adj. Recto, conforme a la moral.

3. m. desus. Persona que estudia o enseña moral.

4. f. Parte de la filosofía que trata de la moral y de las obligaciones del hombre.

5. f. Conjunto de normas morales que rigen la conducta humana. Ética profesional

Será en su quinta acepción donde encontraremos el significado que posee cuando hablamos de ética empresarial, aunque la Real Academia de la Lengua utilice como ejemplo para expresarlo el término ética profesional. Por tanto, para el objeto de nuestro estudio entenderemos la ética como ese conjunto de normas que determina el obrar en términos de bien o mal, de correcto o incorrecto, en concordancia con la sociedad o uno mismo y para el caso concreto de la actuación de las empresas en su función social. 
Al referirnos a la función social de las empresas es obligatorio hablar de la ética empresarial. En concreto, como así hemos convenido, porque a esta última la podemos considerar como el terreno de juego para tratar la complejidad moral en la toma de decisiones. Más aún, si entendemos que la manera en que una empresa alcanza sus objetivos "es dentro de la esfera de la ética" y que, como reflejan Sisk y Sverdlik (1979:71), "los sistemas de valores personales determinan para un individuo, lo que está bien o incorrecto, lo que es bueno o malo, exitoso o fallido, placentero o desagradable, o cualquier otra evaluación bipolar similar".

Es imprescindible analizar, por lo expuesto, la función directiva, en concreto la configuración de las decisiones. Ello se debe a dos razones. La primera porque debemos tener en cuenta que no dejamos de ser animales éticos, o como denominaba Aristóteles, "rex politica". La segunda por la vinculación entre decisiones individuales y repercusiones sociales, en concreto por considerar que "cuando las empresas evalúan decisiones desde una perspectiva ética, debe haber una presunción que favorezca la adopción de cursos de acción que aumenten el bienestar de la sociedad en general" (Hill y Jones, 1996:61).

De hecho, las repercusiones sociales que llevan aparejadas las actividades empresariales son cada vez más tenidas en cuenta a la hora de fijar los comportamientos a futuro como así defiende Mc Coy (1985:87) cuando afirma que "tratar con valores requiere tener en cuenta continuamente el entorno que te rodea, sopesando las diferentes alternativas de acción, equilibrando e integrando responsabilidades contrapuestas, estableciendo prioridades entre objetivos enfrentados, y estableciendo criterios para definir y evaluar la realización de las políticas empresariales. Unido a todo ello va el tratar de articular formas de alcanzar un reflejo ético directa y completamente en los procesos en los que la política de la empresa se articula, implementa y evalúa. Cada vez en mayor medida, las habilidades que tienen que ver con valores como componentes integrales de la consecución de objetivos económicos en la empresa y la concreción de sus políticas se están reconociendo como puntos centrales de una gestión efectiva en una sociedad y un mundo sometidos a rápidos cambios". Así se recoge, por ejemplo, en la encuesta realizada por The Economist en octubre de 2004, en la que el 85 por ciento de los entrevistados, directivos e inversores institucionales, consideran que la responsabilidad social empresarial es un "aspecto central o importante para la toma de las decisiones de negocio".

Más aún, debemos tener en cuenta que la denominada ética empresarial forma parte de la ética cívica. Las personas que toman las decisiones en las empresas son influidas, y a la vez influyen, en la conformación de los valores morales de su comunidad. Existe una retroalimentación constante entre individuo y sociedad en la conformación de una conciencia moral alcanzada por el conjunto de la comunidad, es decir, los principios y valores universales mínimos compartidos para un espacio y tiempo concretos.

Llegado a este punto nos encontramos con planteamientos opuestos en la configuración de la toma de decisiones por parte de la dirección de una empresa. En concreto, 
ante lo que podríamos denominar la posición clásica frente a la posición socioeconómica (Robbins y Coulter, 2000:142). La primera defendería que la orientación de la posición directiva tiene como único objetivo la maximización de los beneficios financieros, mientras que la segunda incorpora elementos externos al devenir propio empresarial, así podemos señalar, entre otros, el propio bienestar de la sociedad. Las dos figuras preponderantes de estas propuestas serán Friedman y Freeman, respectivamente

Antes de pasar a analizar de forma más concreta estas dos visiones debemos pararnos a reflexionar brevemente sobre otras concepciones de la ética social que han influido en los comportamientos empresariales. Así, citando las palabras de Habermas (1988:75) encontramos que "los clásicos de la teoría social, desde Karl Marx a Max Weber, coincidían en que la estructura de la sociedad burguesa giraba en torno al trabajo abstracto, esto es, un tipo de trabajo básicamente industrial orientado por las leyes del mercado, sometido a las leyes del valor capital y organizado según criterios empresariales. De una producción correctamente organizada tenía que surgir la forma de vida comunitaria de trabajadores libres asociados. La idea de autogestión obrera todavía inspiró los movimientos de protesta de final de los años setenta". Es decir, entendían que el sistema empresarial capitalista llevaba consigo el paso hacia unos medios de producción de gestión colectiva por los propios trabajadores, pasando de la toma de decisión unilateral a otra de orientación colectiva, caso que pueden reflejar las sociedades cooperativas.

Retomando los dos planteamientos a los que hacíamos referencia, encontramos que la posición de Friedman (1970) sobre este tema se corresponde perfectamente a sus planteamientos acerca del papel de las empresas, como así atestiguan sus palabras: "en una empresa libre, en un sistema de propiedad privada, un ejecutivo corporativo es un empleado de los propietarios del negocio. Él tiene una responsabilidad directa ante sus patronos. Esa responsabilidad consiste en dirigir la empresa conforme a sus deseos, los cuales generalmente consistirán en ganar tanto dinero como sea posible conformándose a la vez a las reglas básicas de la sociedad, aquellas incorporadas en las leyes y aquellas incorporadas en las costumbres éticas...En tanto que estas acciones reduzcan los rendimientos para los inversionistas, él estará gastando el dinero de ellos. En tanto que sus acciones eleven el precio a los clientes, él estará gastando el dinero de los clientes".

Enfoque que también es defendido, entre otros, por Theodore Levitt (1958) por cuanto entiende que la maximización de beneficio debe continuar siendo para la empresa el objetivo preferente, y esto tanto en la teoría como en la práctica. Esta idea, además, se fortalece por la propia dinámica en la configuración hasta ahora de los estímulos para la gerencia si convenimos que ha sido mucho más fácil diseñar incentivos para que los gestores maximicen beneficios que hacerlo de forma que estas mismas personas asuman en sus decisiones las preferencias de los grupos de interés de la empresa.

Frente a la simplificación en la conformación de la toma de decisiones del planteamiento clásico, se encuentra lo que hemos denominado la posición socioeconómica. 
Ésta, defendida por Freeman (1984), se asienta en la convicción de que los gestores actúan siempre en función de los objetivos de un tercero, ya sean los accionistas o los grupos de interés. Más en concreto, argumenta que los directivos, por lo general, responden a cuestiones de los grupos con los que tratan al hacer negocios, particularmente se interesan por las demandas y preocupaciones de sus grupos de interés. Visión que es compartida, entre otros, por Calrkson (1985) y Koontz y Weihrich (1998:62). Éstos últimos han afirmado que "los administradores responderán a los valores aprobados por la sociedad y darán prioridad a los tenidos en mayor estima". Por estos argumentos, la posición tradicional en la ética empresarial se ve relegada, por cuanto se considera que el desarrollo y reforzamiento de la ética empresarial es necesaria para lograr la viabilidad del proyecto empresarial.

La ética empresarial se convierte en el nexo de unión entre la idoneidad de las decisiones y la respuesta de la sociedad civil, principalmente si convenimos que ésta última ha adquirido un protagonismo mayor del que hasta ahora se percibía "demandando directamente de las empresas que consideren las implicaciones sociales de sus actuaciones económica, tanto en el interno mediante los trabajadores y sindicatos, como en su externo a través de las Administraciones Públicas y organizaciones del tercer sector, entre otros. La presión social y la legislación sobre temas sociales y medioambientales constituyen restricciones a la función maximizadora de beneficios" (Cuervo, 2005: 20). Por tanto, las organizaciones que no tengan presente esto, por cuanto sus actuaciones causan repercusiones en el sistema social, se verían amenazadas por lo que Davis (1979: 316) llama la "Ley de Hierro de la Responsabilidad Social". Es decir, "a largo plazo, aquellas instituciones que no ejerzan el poder en concordancia con las expectativas de la sociedad, tienden a perderlo". La credibilidad social se ve resentida cuando las organizaciones toman decisiones que no llevan consigo una justificación en línea con las demandas sociales. Por ejemplo, cuando una empresa que da beneficios despide a un número importante de trabajadores la sociedad exige con más empeño las razones de esta decisión.

Siguiendo este razonamiento la empresa deja de ser exclusivamente una actividad privada en la medida que cada vez más se ve obligada a depender de la implicación, cooperación y respaldo de sus diversos grupos de intereses. La implicación de las organizaciones en su entorno, así como los beneficios que socialmente pueden aportar, se traducen a la larga en un refuerzo de su imagen en el mercado. Muchos directivos, principalmente de las multinacionales, intentan acomodar sus decisiones al marco de la responsabilidad social empresarial como mecanismo para reducir el riesgo de confrontaciones innecesarias en el ámbito social. Ahora bien, debido a la enorme cantidad de variables que engloban las exigencias sociales, nos podemos encontrar que gerentes que pretenden cumplir con esta responsabilidad no tienen otra alternativa que confiar en sus propias ideas, intereses y valores; o seguir ciertas directrices generales acerca de la dinámica de los valores sociales y de las nuevas y crecientes expectativas de parte de 
la comunidad. De hecho, un primer paso ha sido adoptar en muchas de estas empresas códigos de conducta que vienen a marcar los comportamientos internos en la tomas de decisiones, fomentando la introducción de niveles de protección más elevados en la vinculación de estas decisiones con la previsible respuesta social.

La toma de decisiones que no llegue a interpretar correctamente las demandas de la sociedad puede dar como resultado la aparición de inconvenientes para conseguir los objetivos de rentabilidad económica marcados por cualquier empresa. Un buen ejemplo de lo dicho son las prácticas llegadas a cabo por Infact, que desde 1977 vigila las actuaciones de las multinacionales para evitar situaciones abusivas. Así ocurrió en su boicot de siete años, hasta 1984, a los productos de la multinacional Nestlé, la mayor empresa de alimentación del mundo, por considerar que la empresa ponía en riesgo la vida infantil en países poco desarrollados con sus campañas publicitarias. Después de este tiempo de continua presión y ante la erosión que estaba sufriendo la imagen de la compañía, representantes de la empresa tuvieron que negociar con Infact para lograr el fin del boicot. La concienciación sobre los problemas de las campañas publicitarias llevadas a cabo por la multinacional suiza trajo consigo, además, la reforma de parte de la Organización Mundial de la Salud y del Código internacional para la publicidad de sustitutos de la leche materna.

Es en base a este principio de relación entre decisiones empresariales y respuesta de la sociedad desde donde se han venido desarrollando nuevos enfoques sobre lo que es la gestión empresarial socialmente responsable. Así aparecen nuevos conceptos para la satisfactoria administración de empresas, como el caso concreto de la denominada ciudadanía corporativa o empresarial que aparece en los años noventa del pasado siglo. No obstante, debemos señalar que la principal presión que ha sufrido la dirección de las empresas ha venido de la tradicional lucha de intereses con sus trabajadores, de tal forma que el primer principio ético de una empresa se debe situar en el comportamiento con sus trabajadores. De hecho, podemos encontrar ejemplos claros de una mayor vinculación entre decisiones y participación de los trabajadores en la gestión empresarial, como el denominado modelo "Toyota" o las co-dirección de las grandes multinacionales alemanas.

La ciudadanía empresarial nace como un término que intenta albergar las prácticas que delimitan el comportamiento responsable de las empresas en su relación con la sociedad desde una práctica proactiva e integral. Su teorización se basa en el modelo de los stakeholders propuesto por Freeman y en la concepción de que la empresa a la hora de diseñar e implementar sus estrategias deber tener en cuenta los efectos externos de las mismas sobre todos los agentes sociales implicados en su actividad.

Autores como Logan, Roy y Regelbrugge (1997: 344) afirman que la ciudadanía corporativa posee una realidad muy extensa ya que puede variar entre los mínimos deberes legales y éticos exigidos por la comunidad, hasta la implementación de un comportamiento responsable y proactivo por el otro. Así se desprende de sus palabras: "como mínimo, la ciudadanía corporativa significa adherirse a las leyes, regulaciones y prácticas de negocio aceptadas en el lugar donde opera la compañía. Según una 
interpretación más amplia: son las formas de conducta de la empresa que reflejan un comportamiento responsable y proactivo, tanto en los negocios como en el trato con todos sus integrantes y con respecto a las comunidades, sociedad y medio ambiente".

Por tanto, podemos convenir que la ciudadanía corporativa se considera un proceso mediante el cual una compañía desarrolla y administra las relaciones con sus grupos de interés, en base a la supuesta correspondencia entre relaciones-rentabilidad económica (Waddock y Smith, 2000). Se trata, por ello, de conseguir una corresponsabilidad con todos los afectados por la actividad de la empresa, tomando conciencia de las implicaciones sociales y humanas de las mismas, desarrollando políticas, procedimientos y procesos que respeten las demandas de cada grupo de interés.

Llegados a este punto en la conceptualización de las prácticas sociales de las empresas, no es extraño encontrar autores que llegan a enumerar cuales son las principales responsabilidades éticas de las organizaciones con sus grupos de interés y con la comunidad en su conjunto. Para Bestratén y Puyol (2004: 644), por citar un caso concreto, estas obligaciones serían las siguientes:

- "Servir a la sociedad con productos útiles y en condiciones justas.

- Crear riqueza de la manera más eficaz posible.

- Respetar los derechos humanos con unas condiciones de trabajos dignas que favorezcan la seguridad y salud laboral y el desarrollo humano y profesional de los trabajadores.

- Procurar la continuidad de la empresa y, si es posible, lograr un crecimiento razonable.

- Respetar el medio ambiente evitando en lo posible cualquier tipo de contaminación, minimizando la generación de residuos y racionalizando el uso de los recursos naturales y energéticos.

- Cumplir con rigor las leyes, reglamentos, normas y costumbres, respetando los legítimos contratos y compromisos adquiridos.

- Procurar la distribución equitativa de la riqueza generada".

Estos planteamientos refuerzan la idea que las empresas no sólo tienen una función económica, sino una función social y que las decisiones empresariales deben amoldarse a los interese sociales. En un mercado global, competitivo y de cada vez más información, como en el que estamos inmersos, conseguir el reconocimiento social y la credibilidad de sus actividades se ha convertido en pieza clave para la legitimación de las empresas. Enfoque que se ve reforzado por el gran número de estudios publicados sobre el tema, y el hecho de que la gran mayoría de las multinacionales y cada vez más empresas de tamaño medio, dedican un apartado en sus memorias anuales, aunque este pueda considerarse más o menos importante, a explicar sus actuaciones y proyecto de futuro en estos ámbitos a sus grupos de interés. Tradicionalmente las prácticas económicas, que hacían referencia a aspectos privados de las organizaciones, se reflejaban en la documentación que con carácter periódico y obligatorio era requerida por los 
organismos reguladores. En la actualidad, junto con el informe económico anual, se especifica la información sobre el cumplimiento de los objetivos sociales, incluso se llega a publicar por separado. La edición independiente puede ser reflejo de una concepción diferente en la estrategia empresarial. Esto es, bien porque se considera de tal importancia satisfacer las necesidades informativas de los grupos de interés que se asigna un espacio propio, o por el contrario, que la información económica y social no posee una conexión directa y, por ello, la elaboración de informes separados es debido a la supuesta falta de relación entre ambas.

Los grupos de interés, a los que la empresa debe de alguna manera rendir cuentas si pretende asegurar su continuidad a largo plazo, serían sus accionistas, socios, los propios trabajadores y sus organizaciones sindicales, clientes y consumidores, proveedores, organizaciones sociales y medios de comunicación, y a través de estos últimos el conjunto de la ciudadanía.

Muestra de la importancia que ha adquirido la comunicación de las empresas con sus grupos de interés es la incorporación, en los últimos años, de conceptos como sostenibilidad social y responsabilidad social dentro de sus informes. De hecho se ha generalizado la presencia junto a las memorias financieras de documentación específica que refleja las prácticas sociales y de sostenibilidad. Está práctica se ha visto acentuada tras la aparición a comienzos de esta década de casos como los de ENRON, WorldCom o AHOLD, en la expectativa de evitar situaciones perjudiciales que pongan en riesgo la confianza de los distintos agentes en el desempeño de los mercados y, por tanto, en su satisfactorio desarrollo. Las empresas por esta vía pretenden alejarse de la imagen de aquellas otras que no responden a los valores sociales, pudiendo incluso lastrar así al conjunto de un sector concreto, pero sobretodo porque se desprende que cada vez con más intensidad los consumidores reaccionan de forma positiva ante los productos y servicios de aquellas empresas que mantienen un compromiso social, hasta el punto de llegar a cambiar de preferencias, caso de la introducción en algunas grandes cadenas de distribución de alimentos con conocidos productos de "comercio justo". Por esta razón, las empresas que realicen actuaciones consideradas socialmente responsables, o incluso aunque no las lleven a cabo pero intenten dar esta imagen, están dedicando parte de sus recursos a la publicidad de las mismas.

Comprobamos cómo las prácticas empresariales éticas se expresan desde dos parámetros interrelacionados. Por una parte, la función interna de la compañía incorporando a sus procesos las exigencias sociales en base a la responsabilidad que se les entiende. Por la otra, la externa que implica dar valor en el mercado a esos nuevos atributos, mediante estrategias adecuadas de comunicación. De aquí deriva el reforzamiento en la administración empresarial de conceptos como el marketing social, el marketing social corporativo y el marketing con causa.

Siguiendo la definición más aceptada propuesta por Kotler y Zalltman (1971:10), “el marketing social es el diseño, implementación y control de programas pensados 
para influir en la aceptación de ideas sociables, implicando consideraciones de planificación de productos, precio, comunicación, distribución e investigación de marketing". De ellos será el marketing con causa el que pretende de una forma directa encontrar una posición de ventaja para captar y retener clientes, en un sistema de una fuerte competencia global, a través del valor de marca, de hecho según Pringle y Thompson (1999: 3) es "una herramienta estratégica de marketing y posicionamiento, que vincula una empresa o su marca a una causa social de interés, en una relación de beneficio mutuo", encontrando las imbricaciones entre el valor de marca, la responsabilidad social, los grupos de interés y la primacía del consumidor.

Ello se debe a que, según estos mismos autores, "las marcas son consideradas por los consumidores como una promesa, una expectativa de valor más allá de lo que les ofrecen. Son más que el nombre, logotipo, colores, embalaje y diseño, creados por los medios de comunicación para firmar un compromiso. Las marcas han evolucionado de un enfoque racional, al emocional, y finalmente hacia una perspectiva espiritual o ética. La perspectiva espiritual o ética basada en creencias y valores que las instituciones añaden a su marca, como un reflejo de su responsabilidad social corporativa" (Pringle y Thompson, 1999: 68). Las empresas pretenden asociar sus marcas, y por ende su imagen como compañía, a causas que interrelacionen sus valores de negocio con los asumidos socialmente, bien sea de forma independiente o con el respaldo de algunos grupos sociales, como en el caso del certificado de sostenibilidad forestal, más conocido por sus siglas en inglés, FSC (Forest Stewardship Council).

Por estos motivos, la ética empresarial ha pasado de ser un fin ideal e incondicional a ser un medio para el beneficio económico. Nos encontramos, en el actual sistema de competencia global, ante una de las fuentes que las empresas están explotando para maximizar sus beneficios. Se pasa de la concepción de freno para el desarrollo del negocio a un instrumento estratégico de gestión empresarial que permitiría un mejor desempeño económico, aumentando de competitividad y, por lo tanto, su valor. Así las grandes multinacionales, principalmente, han adoptado un creciente interés por demostrar un desempeño social y ambientalmente responsable, que se refleja en los criterios de su gobernabilidad corporativa, a través de códigos de buenas prácticas empresariales que contemplan la información a todos sus inversionistas, y que en algunos casos incluye su participación activa en el diseño y discusión del plan de negocios de la empresa. Estos niveles de gestión social, se manifiestan, especialmente, en los llamados índices ético-sociales que en la actualidad engloban a las empresas con mayores compromisos en este ámbito.

Ante la presión social cada vez se hace más necesario potenciar la ética empresarial como factor clave para implementar en las organizaciones una correcta visión de responsabilidad. De hecho, esta responsabilidad con la comunidad pasa a tener así una relevancia estratégica cuando las empresas destinan recursos a una causa social, no como donación o simple filantropía, sino como una apuesta de posicionamiento institucional o de marca. 
Aparece, por tanto, el enfoque que defiende la concepción estratégica de la ética empresarial, superando el enfoque meramente instrumental, por cuanto no sólo se proyectan beneficios económicos sino también sociales, en forma de demanda de los diferentes grupos de interés, reconociendo en la gestión de los mismos la creación de valor a largo plazo.

Por ello, dentro de la ética empresarial la responsabilidad social se llega a plantear como una estrategia social que debe estar ligada con la misión, visión y valores de la empresa, integrándola a la estrategia global de la organización, de forma que los beneficios se hagan patentes a nivel económico y social, garantizando la competitividad a largo plazo de la empresa y su desarrollo sostenible en el tiempo. Es decir, se incorpore a la cadena de producción o a la creación de servicios, así como a la cadena de valor y en la gestión de las relaciones con los diversos grupos de la empresa. En otras palabras: "un aumento de la democracia interna en las empresas no sólo puede garantizar un entorno laboral más agradable, sino también más innovador y, con ello, una sociedad más innovadora en su conjunto" (Stiglitz, 2008)

Se revierte el concepto del cumplimiento de las exigencias sociales como una oportunidad a corto plazo que genera un retorno puntual o, simplemente, un imperativo moral que la empresa se ve obligada a seguir; para dar paso a una responsabilidad social de la empresa como parte estratégica, con un alcance a largo plazo. Idea que se ve reforzada por publicaciones como las de Burke y Logsdon (1996), Mac Williams y Siegel (2001) y Dentchev (2004), donde se argumenta que un mayor output social y una creación de valor para la empresa puede obtenerse de un enfoque estratégico más que por uno filantrópico o altruísta desarrollan para ello marcos teóricos e incluso estudios empíricos para apoyar esta idea.

Este posicionamiento responde a la concepción sobre la responsabilidad económica de la empresa, por cuento la maximización del beneficio empresarial está íntimamente ligada con la creación de valor para el accionista. Con la maximización del beneficio como objetivo prioritario de la empresa volvemos a la teoría económica neoclásica, si bien debemos recordar que Clark (1957) señaló que la maximización de los beneficios era un concepto difícil de definir, dejando espacio para la incorporación en él de la buena ciudadanía.

No será hasta mediados de la década de los 70 del pasado siglo cuando empiecen a analizarse en la práctica las vinculaciones entre el desempeño socialmente responsable y la rentabilidad financiera de las empresas (Burke y Logsdon, 1996). A día de hoy no existe un consenso sobre la correlación entre estas variables. Mientras que el estudio de Waddock y Graves (1997) muestra una vinculación positiva, el análisis de Wright y Ferris (1997) lo hace de forma negativa. Por otra parte en el trabajo de Aupperle, Carroll y Hatfield (1985) no identifican ningún tipo de nexo entre ambas cuestiones.

Ante esta situación algunos autores (Starik y Carroll, 1990) consideran que el problema está en la conceptualización de los que se entiende por la responsabilidad so- 
cial ejercida por las empresas, a lo que se añade la enorme dificultad para encontrar instrumentos de medición adecuados, como así ocurre con otros términos de reciente sistematización, como es el capital social. Junto a estas debilidades propias de la investigación empírica se añade la problemática de encontrar datos homogéneos en los supuestos comportamientos responsables de las empresas.

Es por esta razón que los últimos intentos por determinar la correlación entre desempeñó social y rentabilidad financiera se enmarquen (Burke y Longsdon, 1996) en el camino de definir las estrategias a largo plazo en contra posición a los beneficios cortoplacistas. Prueba de ello es que las empresas, en especial las multinacionales, coinciden en considerar el mercado global no solo en un fenómeno comercial, sino también en su perfil de transformador social, pues, cada vez más, se ven obligadas por la sociedad a mantenerse en un marco de desarrollo sostenible, el cual se sustenta en un crecimiento económico que no olvide un mayor posicionamiento ético para abordar las exigencias sociales, tales como los derechos humanos y laborales, la protección del medio ambiente, la igualdad de trato, la transparencia en la información, etc.

No obstante, como puntualiza Friedman, todo puede quedar en un mero ejercicio de marketing publicitario o, peor aún, en una justificación de situaciones moralmente injustificables, si no somos capaces de definir, en primer lugar, en qué consiste esta responsabilidad, cuáles son sus márgenes; $\mathrm{y}$, en segundo lugar, cómo podemos medirla y evaluarla.

Así, podemos llegar a distinguir aquellas estrategias empresariales que permitiendo la generación de beneficios son etiquetadas como actuaciones socialmente responsables. Entre otras, citar las externalidades que produce una empresa que afectan al valor del bien para los clientes, como es el caso de un hotel que invierte en la protección de la naturaleza cuando es uno de sus principales atractivos turísticos; comportamientos altruistas con sus clientes para no perder el valor de marca, como por ejemplo determinadas marcas de vehículos de lujo que cuando detectan un problema en alguno de sus productos realizan revisiones gratuitas; o, como en el caso de prácticas beneficiosas para la comunidad en precios y servicios para evitar la intervención de las instituciones regulatorias de la competencia (caso de Microsoft).

La reflexión a la que nos conducen los debates recientes sobre llamada ética empresarial es si todas las decisiones adoptadas por una empresa, que en teoría pretenden responder a las demandas sociales, pueden ser consideradas como actuaciones de responsabilidad social, puesto que como hemos visto existen estrategias gerenciales que permiten hacer compatible la maximización de beneficios y el cumplimiento de las exigencias de los grupos de interés. De hecho, en muchos casos el desarrollo de programas de responsabilidad empresarial puede mejorar el bienestar social, a la vez que logran beneficios en la empresa a través de mejoras de competitividad.

El problema reside, siguiendo a Friedman, en la imprecisión del término de responsabilidad social empresarial, pues nos encontramos ante un concepto que no se muestra 
estático, preciso y definido, sino como un ideal que recogería todas las expectativas, tanto de tipo económico, legal, ético y discrecional, que la sociedad se ha forjado con respecto a las organizaciones empresariales en un momento determinado de su acontecer histórico.

Es por ello, que para poder llegar a analizar socialmente las prácticas concretas que las empresas realizan en base a una supuesta responsabilidad moral, es necesario completar el análisis con una interpretación concreta en su conceptualización.

\section{La responsabilidad de las decisiones empresariales con la sociedad.}

Conseguir el logro de encontrar la definición precisa del término responsabilidad social empresarial permitiría delimitar, de alguna manera, el alcance de las acciones de una empresa sujetas a esta dinámica y, al mismo tiempo, entrever, si lo hubiese, el aspecto de obligación moral de la misma.

Para emprender ordenadamente esta búsqueda quizá la mejor aproximación sea la semántica, recordando, además, que todo concepto está sujeto a dos dimensiones: la comprensión y la extensión. Tratemos, pues, de definir la responsabilidad social empresarial desde el significado de sus componentes.

En lo que se refiere a la cuestión semántica, la palabra responsabilidad proviene de la unión del verbo latino "respondeo" que significa responder y del sufijo "abilis" como expresión de la facultad de ser capaz. Por tanto, desde el punto de vista etimológico podemos entender la responsabilidad como la capacidad de responder, es decir de la defensa de una acción previa ante terceros. La consideración de responsabilidad sugiere tres cuestiones: la posibilidad de elección libre, la realización de una acción y la justificación de la misma ante otros. Ello es debido a que la exigencia de responsabilidad no es posible cuando no existen alternativas de elección. Así, para la Real Academia de la Lengua responsabilidad es "poner atención en lo que se hace o se decide". La concepción etimológica es superada en la práctica añadiéndose la carga moral, por cuanto entendemos la obligatoriedad de responder de forma satisfactoria, hablando de responsabilidad casi exclusivamente cuando las respuestas tienden a ser las mejores. Prueba de este particular la encontramos en el artículo 1902 del Código Civil donde se recoge, al referirse a responsabilidad "el que por acción y omisión causa daño a otro, interviniendo culpa o negligencia, está obligado a reparar el daño causado".

La responsabilidad en la actualidad se configura como base del entramado social para la consecución de objetivos comunes, como así defienden los teóricos del capital social. Es aquí donde encontramos las características que conforman el concepto: alternativas de elección, acción libre y previsión de las consecuencias. Será el resultado final el que nos ponga en relación con otros, siendo por tanto el cumplimiento hacia el otro el que genera esta responsabilidad, además de la confianza recíproca.

Recordemos que la responsabilidad como cualidad es parte de la virtud de la justicia. De aquí se deriva que la responsabilidad moral de cada persona esté compuesta 
tanto por derechos como por obligaciones. Si toda acción libre puede ser considerada como una acción moral es entonces cuando podemos hablar del vínculo entre responsabilidad y moralidad, más en concreto de la citada responsabilidad moral. Caso concreto de la mayoría de las decisiones empresariales, ya que al fin económico se le añade las posibles consecuencias derivadas a terceros. Por lo anterior las decisiones empresariales no pueden considerarse como conductas amorales. A todo ello se añade que las iniciativas empresariales no corresponden a personas individuales sino a posicionamientos colectivos de actuación, por cuanto se habla de responsabilidad empresarial en su conjunto y no de responsabilidad personal dentro de las empresas. La empresa responde como institución.

Así, al hablar de responsabilidad empresarial nos encontramos que los otros es la sociedad, de aquí se deriva la configuración del concepto de responsabilidad social empresarial. Si bien, como apreciábamos al hablar de la nueva función de las empresas en la sociedad, la responsabilidad empresarial se muestra como la base del impacto socioeconómico de la empresa en las comunidades en las que opera. Es decir, la sociedad a la que se refiere la responsabilidad social empresarial exige de determinadas características en el comportamiento de las empresas hacia las nuevas exigencias sociales referenciadas en el caso concreto de sus grupos de interés.

En lo que respecta al tercero de los términos que componen el concepto de responsabilidad social empresarial, debemos diferenciarlo de otras acepciones que vienen siendo utilizadas en la actualidad, nos estamos refiriendo al término corporativa. La corporación debe ser entendida de forma más amplia que la organización empresarial privada tradicional, por cuanto engloba a todo tipo de instituciones que de alguna forma generan impacto socioeconómico en la comunidad donde desarrollan su actividad. Nosotros nos hemos centrado en el término concreto referido a la empresa desde el punto de vista de la teoría económica, como organización privada cuyo objetivo es lograr beneficios. Por ello, entendemos la empresa para este estudio como sujeta a un marco democrático de libre mercado, pues desde el momento en que toda empresa puede hacer las cosas de una u otra forma, se concibe que dispone de un espacio de libertad del que debe dar razón, del que es responsable. La empresa a la que se refiere el concepto de responsabilidad social empresarial estaría, en un primer momento, compuesta por tres dimensiones: ética, administrativo-económica y jurídico-social.

La dimensión ética debe ser entendida en la suma de dos cuestiones. La primera en cuanto a la virtud moral de sus directivos, la segunda en cuanto a la cultura y las estructuras organizativas de las que se componen. La variable ética es la forma de entender tanto desde el punto de vista individual como colectivo la influencia de las decisiones en el externo de la propia organización.

La dimensión administrativa financiera es, posiblemente, la característica de más fácil percepción de las que en la actualidad hemos dotado a la empresa. A la visión tradicional de entender la finalidad de la empresa como el logro de beneficios, bajo un marco económico y legal determinado, se debe unir su relación con otros agentes económicos y sociales. 
Por último, la dimensión jurídico-social se entiende como el sistema por el cual se otorga a la empresa y sus grupos de interés un marco de relaciones previsible, compuesto por derechos y deberes.

Por todo ello, se puede entender la responsabilidad social empresarial como el componente moral en la actividad de la empresa desde el punto de vista de la sociedad y respetando la autonomía en la toma de decisiones. En palabras de Habermas (1985:116) "una institución es responsable cuando las decisiones, acciones y políticas que adopta, así como las consecuencias y efectos de las mismas respecto a los intereses en juego, pudieran ser aceptadas por todo los implicados y/o afectados presentes y futuros en un diálogo abierto en condiciones simétricas de participación". Así, puede decirse que es la obligación ética o moral, voluntariamente aceptada por la empresa como institución hacia la sociedad en conjunto, en reconocimiento y satisfacción de sus demandas o en reparación de los daños que puedan haberle sido causados a ésta en sus personas o en su patrimonio común por la actividad empresarial.

\section{Bibliografía}

Aristóteles (1980): "Ética Nicomaquea”, I, 2, siglo IV a. C.; en la traducción de Ross, p.2, citado por A. Sen (1989), Sobre Ética y Economia, p. 22, Madrid, Alianza Editorial.

Arrow, K.J. (1974): Elección social y valores individuales. Madrid, Ministerio de Economía y Hacienda.

Aupperle, K., A. Carroll \& J. Hatfield (1985): "An empirical examination of the relationship between corporate social responsability and profitability", Academy of management Journal, 28, pp. 446-463.

Bestratén, M. y L. Pujol (2004): Responsabilidad social de las empresas (II): tipos de responsabilidades y plan de actuación, España, INSHT, Ministerio de Trabajo y Asuntos Sociales.

BuRKe, L. \& J.M. LogSDon (1996): "How corporative social responsability pays off”, Long Range Planning, 29, pp. 495-502.

CAlrkson, M. (1995): "A stakeholder Framework for analysing and evaluating corporative social performance", Academy of Management Journal, 20 (1), pp. 92-117.

Clark, J.M. (1957): Economic institutions and human welfare, New York, Alfred A. Knopf.

Cuervo, A. (2005): "La maximización del valor de los accionistas versus la responsabilidad social corporativa, ¿compatibilidad?”, Economistas,106, pp. 13-21.

DAvIS, K. (1979): "The case for and against Business assumption of social responsabilities", Academy Journal, 16, pp. 312-322.

Dentchev, N.A. (2004): "Corporative social performance as a Business strategy", Journal of Business Ethics, 55, pp. 397-412. 
EsTAPÉ, F. (1990): Introducción al pensamiento económico. Una perspectiva económica, Madrid, Espasa-Calpe.

Freeman, R.E. (1984): Strategic Management: A Stakeholder Approach, Boston, Pitman Publishing Inc.

Friedman, M. (1970): The New York Times Magazine, 13 Septiembre.

Habermas, J. (1985): Conciencia moral y acción comunitaria, Barcelona, Península.

Habermas, J. (1988): "La crisis del Estado de Bienestar y el agotamiento de las energías utópicas", en Ensayos Políticos, Madrid, Ediciones Península.

Herskovits, M.J. (1952): "Economic Anthropology. A study of Comparative Economy", Nueva York: Alfred A. Knof, citado en R. Tamames (1975), Fundamentos de Estructura Económica, Madrid, Alianza Editorial, p.55.

Hill, C.W.L. y T.M. Jones (1996): Administración Estratégica, un enfoque integrado, $3^{\mathrm{a}}$ edición, Colombia, McGrawHill.

Koontz, H. y H. WeILHRICH (1998): Administración Estratégica, un enfoque integrado, $3^{\mathrm{a}}$ edición, México, McGrawHill.

KotLer, P. \& G. Zaltman (1971): "Social marketing: an approach to planned social change", Journal of Marketing, 35, pp. 9-12.

Levitt, T. (1958): The Dangers of Social Responsibility, Harvard Business Review, Sept-Oct.

Lewis, W.A. (1958): Teoría del desarrollo económico, México, Fondo de Cultura Económica.

Logan, D., D. Roy \& L. Regelbrugge (1997): Global Corporative Citisenship-Rationale and Strategies, Washington DC: The Hitachi Fundation, en C. Marsden and J. Andriof, (1998), Towards an Understanding of Corporative Citizenship and How to Influence it, Citizenship Studies 2, pp. 329-352.

Mac Williams, A., \& D. Siegel (2001): "Corporate social responsability: a theory of the firm perspective", Academy of Management Review, 26, pp.117-127.

McCoy, C.S. (1985): Management of Values: The ethical difference in corporative policy and performance, Marshfield, Mass, Pitman.

Pringle, H. \& M. Thompson (1999): How cause related marketing builds Brands, Chichester, Wiley.

Robbins, S.P. y M. Coulter (2000) : Administración, 6a Edición, México, Prentice Hall.

SEn, A. (1989): Sobre ética y Economía, Madrid, Alianza Editorial.

Sen, A. (2009): "The idea of Justice", Cambridge, Harvard University Press, Resumen de la publicación The Guardian, 13 de Julio de 2009.

Sisk, H. y M. Sverduik (1979): Administración y gerencia de la empresa, EE.UU., SouthWestern Publishing Co.

StARIK, M. \& A. CARRoll (1990): "In search of beneficence: reflections on the connections between firm social and financial performance", Proceding International Association for Business and Societ, Annual Meeting, pp. 1-15. 
StiglitZ, J. (2008): "Conferencia inaugural: del fundamentalismo del mercado hacia una economía más equilibrada", 22 de septiembre de $2008,27^{\circ}$ congreso Internacional del CIRIEC, Sevilla. Centro internacional de investigación e información sobre economía pública, social y cooperativa.

WADDOCK, S. \& S. GRAVES (1997): "Finding the link between stakeholders relations and quality of Management", Business and Society, 16 (3), pp, 250-279.

Waddock, S. \& N. Sмiтн (2000): "Relationships: The Real Chadlenge of Corporative Global Citizenship", Business and Society Review, 105 (1), pp. 47-62.

Wright, P. \& S. FERRIS (1997): "Agency conflict and corporative strategy: the effect of divestment on corporate value", Strategic Management Journal, 18, pp 77-83..

Wright, E.O. (1994): "Reflexionando, una vez más, sobre el concepto de estructura de clases", en J. Carabaña y A. De Francisco (comps.), Teorías contemporáneas de las clases sociales, Madrid, Editorial Pablo Iglesias.. 